Article

\title{
Synthesis of a New Series of Nitrogen/Sulfur Heterocycles by Linking Four Rings: Indole; 1,2,4-Triazole; Pyridazine; and Quinoxaline
}

\author{
Ahmed T. A. Boraei ${ }^{1, *}$, Ahmed A. M. Sarhan ${ }^{2}$, Sammer Yousuf ${ }^{3}$ and Assem Barakat $^{4,5, *(D)}$ \\ 1 Chemistry Department, Faculty of Science, Suez Canal University, Ismailia 41522, Egypt \\ 2 Chemistry Department, Faculty of Science, Arish University, Al-Arish 45511, Egypt; \\ ahmed_sarhan252@yahoo.com \\ 3 H.E.J. Research Institute of Chemistry, International Center for Chemical and Biological Sciences, \\ University of Karachi, Karachi 75270, Pakistan; dr.sammer.yousuf@gmail.com \\ 4 Chemistry Department, College of Science, King Saud University, P.O. Box 2455, Riyadh 11451, Saudi Arabia \\ 5 Chemistry Department, Faculty of Science, Alexandria University, P.O. Box 426, \\ Ibrahimia, Alexandria 21321, Egypt \\ * Correspondence: ahmed_tawfeek83@yahoo.com (A.T.A.B.); ambarakat@ksu.edu.sa (A.B.); \\ Tel.: +966-11467-5901 (A.B.); Fax: +966-11467-5992 (A.B.)
}

Received: 17 December 2019; Accepted: 17 January 2020; Published: 21 January 2020

check for updates

\begin{abstract}
A new series of nitrogen and sulfur heterocyclic systems were efficiently synthesized by linking the following four rings: indole; 1,2,4-triazole; pyridazine; and quinoxaline hybrids. The strength of the acid that catalyzes the condensation of 4-amino-5-(1H-indol-2-yl)-2,4-dihydro-3H-1,2,4-triazole-3-thione 1 with aromatic aldehydes controlled the final product. Reflux in glacial acetic acid yielded Schiff bases 2-6, whereas concentrated $\mathrm{HCl}$ in ethanol resulted in a cyclization product at C-3 of the indole ring to create indolo-triazolo-pyridazinethiones 7-16. This fascinating cyclization approach was applicable with a wide range of aromatic aldehydes to create the target cyclized compounds in excellent yield. Additionally, the coupling of the new indolo-triazolo-pyridazinethiones 7-13 with 2,3-bis(bromomethyl)quinoxaline, as a linker in acetone and $\mathrm{K}_{2} \mathrm{CO}_{3}$, yielded 2,3-bis((5,6-dihydro-14H-indolo[2,3-d]-6-aryl-[1,2,4-triazolo][4,3-b]pyridazin-3 ylsulfanyl)methyl)quinoxalines 19-25 in a high yield. The formation of this new class of heterocyclic compounds in high yields warrants their use for further research. The new compounds were characterized using nuclear magnetic resonance (NMR) and mass spectral analysis. Compound $\mathbf{6}$ was further confirmed by the single crystal $\mathrm{X}$-ray diffraction technique.
\end{abstract}

Keywords: 1,2,4-Triazolel; indole; pyridazine; quinoxalines; linker; annulated heterocycles

\section{Introduction}

Nitrogen-, oxygen-, and sulfur-containing heterocycles are one of the most important compounds found in organic chemistry, as well as in the pharmaceutical industry [1-6]. Among these, 4-amino-1,2,4-triazole-3-thione-based indole scaffolds have drawn considerable attention in the chemical community, including in material science and agrochemical applications. The substituted triazoles have emerged in different pharmaceutical applications including antiproliferative [7], antiviral [8], antimalarial [9], antimicrobial [10], and anticonvulsant agents [11]. Additionally, a few studies have reported their use as inhibitors for metalloenzyme-including ureases [12], dizinc metallo- $\beta$-lactamase [13], the tumor necrosis factor alpha (TNF- $\alpha$ ) converting enzyme [14], ADAM metallopeptidase with thrombospondin type 1 motif 5 (ADAMTS-5) [15], dicopper 
dopamine- $\beta$-hydroxylase inhibitors [16], and also as human carbonic anhydrase enzyme and acetylcholinesterase (AChE) activities [17].

Many triazole-based indoles as a core structure are reported with pharmaceutical targets. Westwell, A.D. et al., independently introduced the synthesis of a set of compounds with a 4-amino-1,2,4-triazole-3-thione-based indole moiety via S-arylation; among these series, they discovered that 3-nitrobenzyl derivative has higher activity in Bc1-2-expressing human cancer cell lines at the BH3 binding pocket with $\left(\mathrm{IC}_{50} \mu \mathrm{M}\right)$ equal to $0.31 \pm 0.03 \mu \mathrm{M}$ for breast (MDA-MB-231), $0.40 \pm 0.07 \mu \mathrm{M}$ for cervical (HeLa), and $0.65 \pm 0.21 \mu \mathrm{M}$ for human leukemia (KG1a), respectively [18]. Another analogue derived from triazole-based indole was reported also by Westwell, A.D. et al., which exhibited anticancer activity against breast MDA-MB-231 and cervical HeLa cell lines; $\mathrm{IC}_{50}=0.91 \pm 0.21 \mu \mathrm{M}$, and $0.25 \pm 0.11 \mu \mathrm{M}$, respectively [19]. In the same area of research, Boraei, A.T.A. et al. explored a set series of compounds including the S-benzylation of a triazole-based indole. Additionally, another set of triazolo-thiadiazepine and triazolo-thiadiazine scaffolds were discovered as potential inhibitors for epidermal growth factor receptor (EGFR) [20,21] (Figure 1).

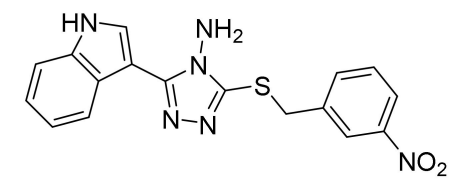

MDA-MB-231 (breast) $\left(\mathrm{IC}_{50}=0.31 \pm 0.03 \mu \mathrm{M}\right)$ Cervical (HeLa) $\left(\mathrm{IC}_{50}=0.4 \pm 0.07 \mu \mathrm{M}\right)$ Human leukaemia (KG1a) $\left(\mathrm{IC}_{50}=0.65 \pm 0.21 \mu \mathrm{M}\right)$

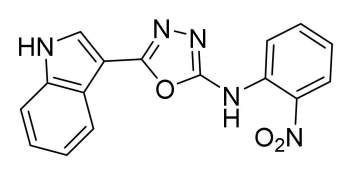

MDA-MB-231 (breast) $\left(\mathrm{IC}_{50}=0.91 \pm 0.21 \mu \mathrm{M}\right)$ Cervical (HeLa) $\left(\mathrm{IC}_{50}=0.25 \pm 0.11 \mu \mathrm{M}\right)$

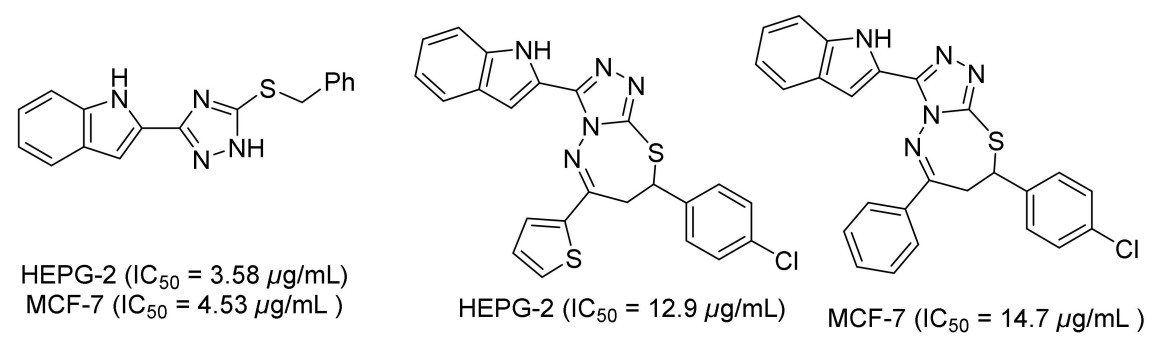

Figure 1. Selected 1,2,4-triazole- indole scaffolds.

A novel fluorophore was derived from a triazole-indole scaffold for a fluorimetric DNA biosensor technique, which was applied for tumor suppressor gene detection and was achieved by Darestani-Farahani, M. et al., in 2018 [22]. Indeed, the indole-triazole Schiff base was synthesized and used as a fluorescent probe for $\mathrm{Al}^{3+}$ ions [23]. This research area reported in the literature regarding indole-triazole hybrids has gained attention from a large number of organic chemists, as well as medicinal chemists.

4-Amino-1,2,4-triazole-3-thione-based indole scaffolds have been used in a large number of organic transformations [24,25], including in metal complexation and in applications for conventional Mizoroki-Heck, and Tsuji-Trost reaction catalysis [26]. Triazole-indole hybrids are commonly reported as synthetic intermediates with hydrazonoyl halides for the synthesis of annulation heterocycles [27]. Gomha, S.M. and Riyadh, S.M. independently applied the microwave technique for the synthesis of a set of compounds with triazole-indole-thiadiazole moieties starting from 4-amino-1,2,4-triazole-3-thione-based indole as a building block, and they explored their potential antimicrobial activity [28]. We have noticed, in the specific reaction of triazole-indole as a core structure with aldehydes, that carbon position number 2 in the indole scaffold plays a crucial rule in chemical transformation [29,30]. Furthermore, novel triazole-indole-oxadiazole compounds were synthesized utilizing triazole-indole hit via ultrasound irradiation, and the synthesized compounds exhibited antimicrobial activity against $E$. coli and $S$. aureus with values of (MIC) between 2 and $8 \mathrm{mg} / \mathrm{mL}$ [31]. One more example derived from an indolo-trizolo scaffold was reported by Diana, P. et al., which showed remarkable antibiofilm activity against $S$. aureus [32]. The quinoxaline scaffold showed interesting biological 
activity [33]. Diana, P. et al., synthesized a new series of aza-isoindolo and isoindolo-azaquinoxaline derivatives which have been discovered to be anticancer agents [34,35].

With these findings mentioned above, here, we reported the synthesis of new multi-nitrogen/sulfur heterocyclic systems by linking the following four rings: Indole; 1,2,4-triazole; pyridazine; and quinoxaline.

\section{Results and Discussion}

\subsection{Synthesis of $\mathbf{2}-\mathbf{1 6}$ and $\mathbf{1 9 - 2 5}$}

The condensation of 4-amino-5-(1H-indol-2-yl)-2,4-dihydro-3H-1,2,4-triazole-3-thione 1 with benzaldehyde, 4-fluorobenzaldehyde, 3-bromobenzaldehyde, $p$-tolualdehyde, and $o$-vanillin in glacial acetic acid yielded the Schiff bases 2-6 in low yield. Alternatively, we carried out the reaction using a stronger acidic medium of concentrated $\mathrm{HCl}$ in ethanol under a reflux condition. Surprisingly, this reaction condition exclusively yielded the cyclized compounds indolo-triazolo-pyridazinethiones $\mathbf{7 - 1 6}$ in excellent yields. The approach was further extended for the substrate scope, and the reaction was performed with ten aromatic aldehydes with different electronic effects: benzaldehyde, 4-fluorobenzaldehyde, 3-bromobenzaldehyde, $p$-tolualdehyde, 2,3,4-trimethoxybenzaldehyde, 4-chlorobenzaldehyde, 4-bromobenzaldehyde, 3,4-dihydroxybenzaldehyde, 4-hydroxy-3-methoxybenzaldehyde, and 2-hydroxy-3-methoxybenzaldehyde (Scheme 1). Compounds 17 and 18 did not occur under the reaction condition.

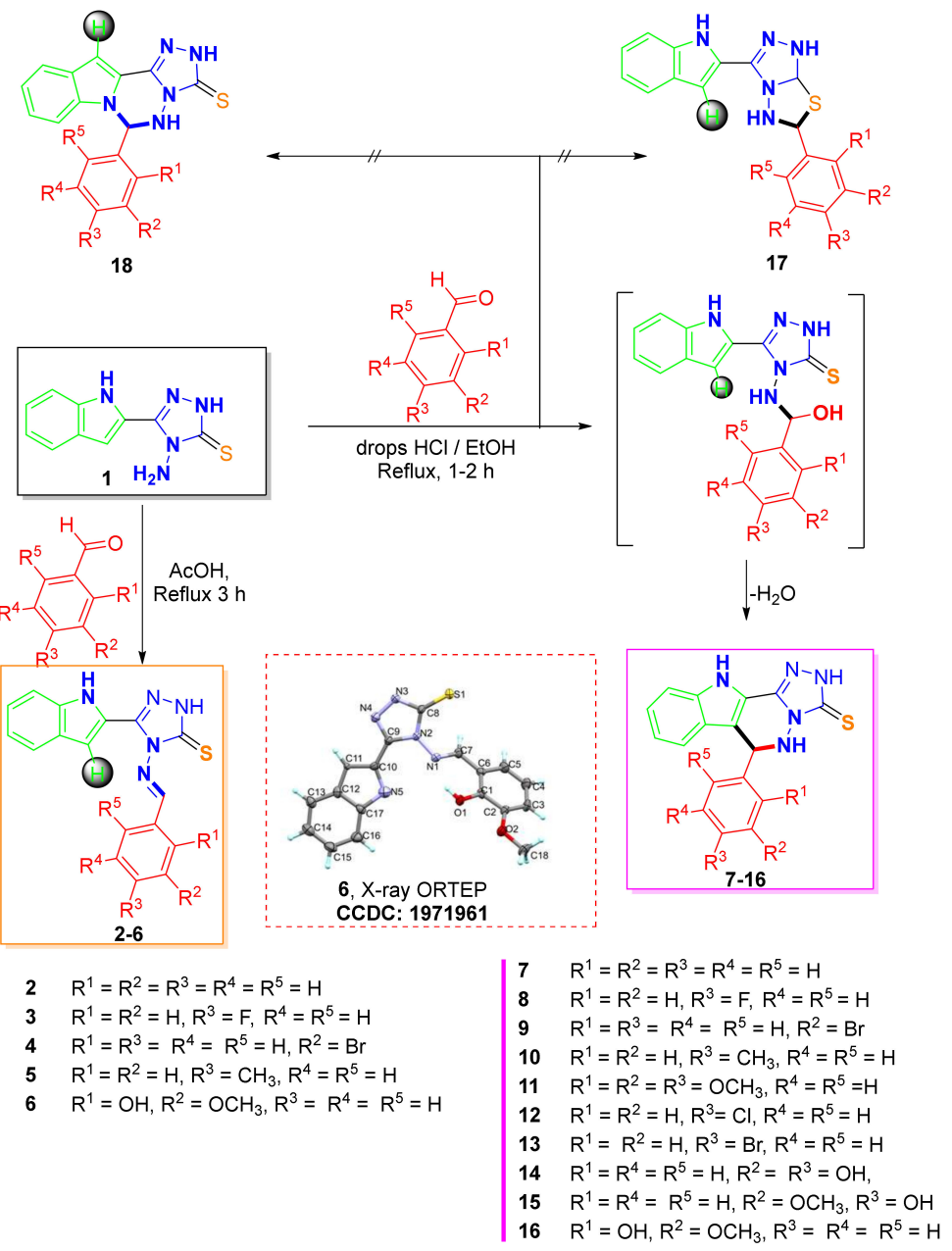

Scheme 1. Synthesis of Schiff bases 2-6 and cyclized indolo-triazolo-pyridazinethiones 7-16. 
The coupling of indolo-triazolo-pyridazinethiones 7-13 with 2,3-bis(bromomethyl)quinoxaline in acetone and $\mathrm{K}_{2} \mathrm{CO}_{3}$ yielded 2,3-bis((5,6-dihydro-14H-indolo[2,3- $d$ ]-6-aryl-[1,2,4-triazolo][4,3-b]pyridazin -3-ylsulfanyl)methyl)quinoxalines 19-25 in excellent yields (Scheme 2).<smiles>[R]c1c([R])c(C2NC(=S)NC2=c2[nH]c3n[nH]c(=S)n23)c2ccccc2c1[R]</smiles>

7-13

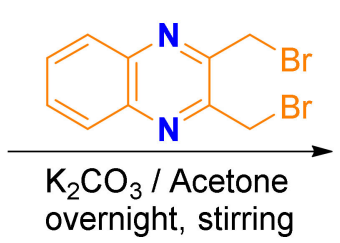

overnight, stirring

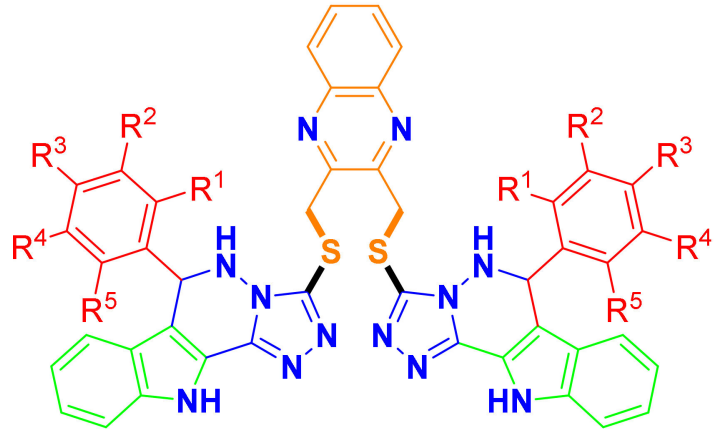

19-25

$$
\begin{array}{ll}
19 & R^{1}=R^{2}=R^{3}=R^{4}=R^{5}=H \\
20 & R^{1}=R^{2}=H, R^{3}=F, R^{4}=R^{5}=H \\
21 & R^{1}=R^{3}=R^{4}=R^{5}=H, R^{2}=B r \\
22 & R^{1}=R^{2}=R^{4}=R^{5}=H, R^{3}=C H_{3}, \\
23 & R^{1}=R^{2}=R^{3}=O C H_{3}, R^{4}=R^{5}=H \\
24 & R^{1}=R^{2}=H, R^{3}=C l, R^{4}=R^{5}=H \\
25 & R^{1}=R^{2}=R^{4}=R^{5}=H, R^{3}=B r
\end{array}
$$

Scheme 2. Coupling of indolo-triazolo-pyridazinethiones 7-13 with 2,3-bis(bromomethyl)quinoxaline.

\subsection{Structural Assignments}

The structural assignments were established based on ${ }^{1} \mathrm{H}$ - and ${ }^{13} \mathrm{C}-\mathrm{NMR}$, and the differentiation between the two isomers 2 and 7 is discussed by comparing their spectra in Figure 2 .

The Schiff base structures 2-6 were assigned from their spectra, which showed the following: The aromatic protons appeared in the range from 7.01 to $8.03 \mathrm{ppm}$, the benzylidene $\mathrm{CH}$ proton appeared as singlet around $9.73 \mathrm{ppm}$, and two $\mathrm{D}_{2} \mathrm{O}$ exchangeable signals were assigned for indole $\mathrm{NH}$ near $11.90 \mathrm{ppm}$, and the second for triazole $\mathrm{NH}$ that found around $14.30 \mathrm{ppm}$. The ${ }^{13} \mathrm{C}-\mathrm{NMR}$ showed only aromatic and thiocarbonyl signals $(\mathrm{C}=\mathrm{S})$ between 105.0 and $167.0 \mathrm{ppm}$ (Figure 2A,B). There was exclusive formation of indolo-triazolo-pyridazinethiones 7-16 whereas, indolo-triazolo-thiadiazole 17 and indolo-triazolo-triazine $\mathbf{1 8}$ did not occur. The structures assignments were deduced from the ${ }^{1} \mathrm{H}-\mathrm{NMR}$, which showed the appearance of pyridazine $\mathrm{CH}$ proton as a doublet around $5.92 \mathrm{ppm}$; then, the aromatic protons and three $\mathrm{D}_{2} \mathrm{O}$ exchangeable signals around 7.01, 12.24, and $13.64 \mathrm{ppm}$ assigned for pyridazine $\mathrm{NH}$, indole $\mathrm{NH}$, and triazole $\mathrm{NH}$ protons, respectively, appeared. ${ }^{13} \mathrm{C}-\mathrm{NMR}$ displayed the pyridazine $\mathrm{CH}$ at $56.13 \mathrm{ppm}$ and the thiocarbonyl signal $(\mathrm{C}=\mathrm{S})$ around $164.73 \mathrm{ppm}$ (Figure 2C,D). In addition, the disappearance of one $\mathrm{CH}$ from the indole signals strongly implied the indolo-triazolo-pyridazinethiones 7-16 structures. Structure 17 was excluded because it should contain the indole $\mathrm{CH}$ and should not contain any thiocarbonyl signal. Structure 18 was also excluded because our structures contain the indole NH. The ${ }^{1} \mathrm{H}-\mathrm{NMR}$ of derivatives 19-25 displayed the methylene protons around $5.00 \mathrm{ppm}$, and the $\mathrm{CH}$ of the pyridazine ring was detected at $5.92 \mathrm{ppm}$. Moreover, two exchangeable protons were detected at $7.00 \mathrm{ppm}$ for $\mathrm{NH}$ pyridazine and $12.36 \mathrm{ppm}$ for the $\mathrm{NH}$ indole. ${ }^{13} \mathrm{C}-\mathrm{NMR}$ demonstrated the methylene carbons around $35.10 \mathrm{ppm}$, and no carbons were detected near to $160.00 \mathrm{ppm}$. The results support the idea that the alkylation of bis(bromomethyl)quinoxaline was performed with sulfur rather than nitrogen. 

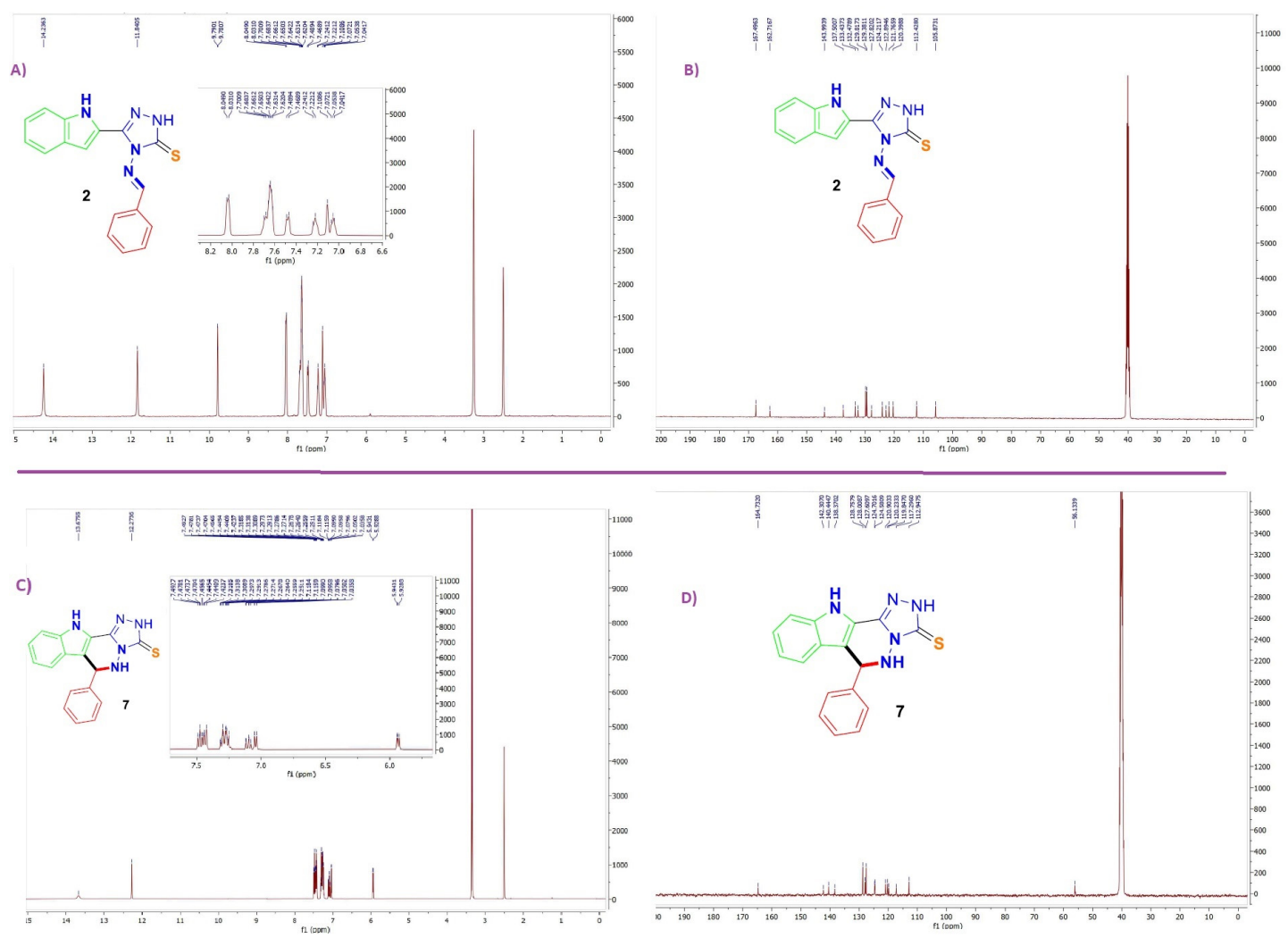

Figure 2. (A) ${ }^{1} \mathrm{H}$ nuclear magnetic resonance (NMR) of 2, (B) ${ }^{13} \mathrm{C}-\mathrm{NMR}$ of $\mathbf{2}$, (C) ${ }^{1} \mathrm{H}-\mathrm{NMR}$ of $\mathbf{7}$, and (D) ${ }^{13} \mathrm{C}-\mathrm{NMR}$ of 7.

\subsection{X-ray Diffraction Analysis of 6}

The structure of $\mathbf{6}$ was determined by single crystal X-ray diffraction (Figure 3). Compound 6 crystallized in a triclinic system and P-1 space group with $Z=2$ and two molecular formula per asymmetric unit (Tables 1 and 2). For simplicity, one of the two-formula units with atom numbering is shown in Figure 3. The lists of the bond distances and angles are collected in Tables S1 and S2 (Supplementary data). The indole moiety is typically a planar system in which the plane passes through the triazole-3-thione, and the aryl moieties are twisted from the plane of the indole plane by $8.91^{\circ}$ and $52.82^{\circ}$, respectively, for the molecular unit shown in Figure 4 . The other molecular unit with higher atom numbering showed similar twists of $6.60^{\circ}$ and $52.98^{\circ}$, respectively [36-38].

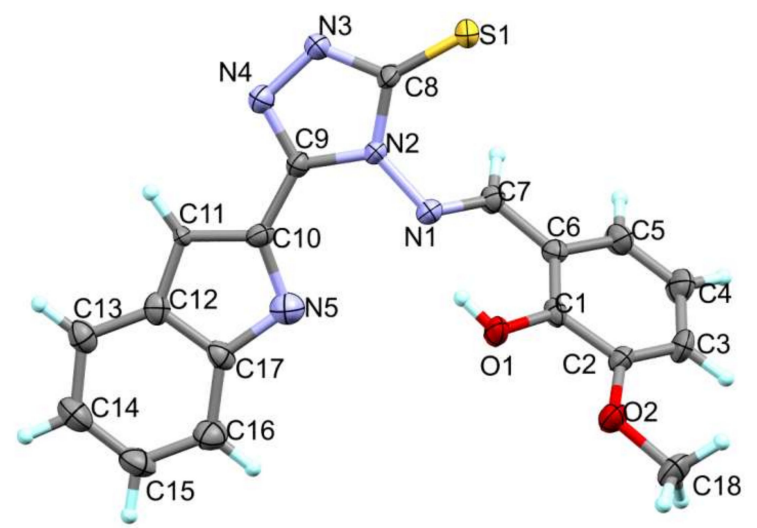

Figure 3. Structure and atom numbering of 6 . 
Table 1. Crystal data for 6 .

\begin{tabular}{cc}
\hline Chemical formula & $\mathrm{C}_{38} \mathrm{H}_{26} \mathrm{~N}_{10} \mathrm{O}_{4} \mathrm{~S}_{2}$ \\
Formula weight & $726.79 \mathrm{~g} / \mathrm{mol}$ \\
Temperature & $104(2) \mathrm{K}$ \\
Wavelength & $1.54178 \AA$ \\
Crystal size & $0.030 \times 0.070 \times 0.130 \mathrm{~mm}$ \\
Crystal habit & Yellow plate \\
Crystal system & Triclinic \\
Space group & $\mathrm{P} \mathrm{-1}$ \\
Unit cell dimensions & $\mathrm{a}=10.1105(6) \AA$ \\
& $\mathrm{b}=13.1764(6) \AA$ \\
Volume & $\mathrm{c}=14.2858(6) \AA$ \\
$\mathrm{Z}$ & $1705.44(15) \AA$ \\
Density (calculated) & 2 \\
Absorption coefficient & $1.415 \mathrm{~g} / \mathrm{cm}^{3}$ \\
$\mathrm{~F}(000)$ & $1.893 \mathrm{~mm}^{-1}$ \\
& 752 \\
\hline
\end{tabular}

Table 2. Data collection and structure refinement for 6 . (RMS, root mean square)

\begin{tabular}{cc}
\hline Theta range for data collection & 3.38 to $68.23^{\circ}$ \\
Index ranges & $-12 \leq \mathrm{h} \leq 12,-15 \leq \mathrm{k} \leq 15,-17 \leq 1 \leq 17$ \\
Reflections collected & 54525 \\
Independent reflections & $6232[\mathrm{R}(\mathrm{int})=0.1173]$ \\
Coverage of independent reflection & $99.9 \%$ \\
Absorption correction & Multi-scan \\
Max. and min. transmission & 0.9470 and 0.7960 \\
Structure solution technique & direct methods \\
Structure solution program & SHELXT $2014 / 5$ (Sheldrick, 2014) \\
Refinement method & Full-matrix least-squares on $\mathrm{F}^{2}$ \\
Refinement program & SHELXL-2017/1 (Sheldrick, 2017) \\
Function minimized & $\Sigma \mathrm{w}^{2}\left(\mathrm{~F}_{\mathrm{o}}{ }^{2}-\mathrm{F}_{\mathrm{c}}{ }^{2}\right)^{2}$ \\
Data/restraints/parameters & $6232 / 0 / 471$ \\
Goodness-of-fit on $\mathrm{F}^{2}$ & 1.085 \\
Final $\mathrm{R}$ indices & 4104 data; \\
& $\mathrm{I}>2 \sigma(\mathrm{I})$ \\
& all data \\
Weighting scheme & $\mathrm{w}=1 /\left[\mathrm{\sigma}^{2}\left(\mathrm{~F}_{\mathrm{o}}{ }^{2}\right)+(0.1000 \mathrm{P})^{2}\right]$ \\
Largest diff. peak and hole & where $\mathrm{P}=\left(\mathrm{F}_{\mathrm{o}}{ }^{2}+2 \mathrm{~F}_{\mathrm{c}}^{2}\right) / 3$ \\
RMS deviation from mean & 1.57 and $-0.52 \mathrm{e}^{-3}$ \\
& $0.110 \mathrm{e} \AA^{-3}$ \\
\hline
\end{tabular}

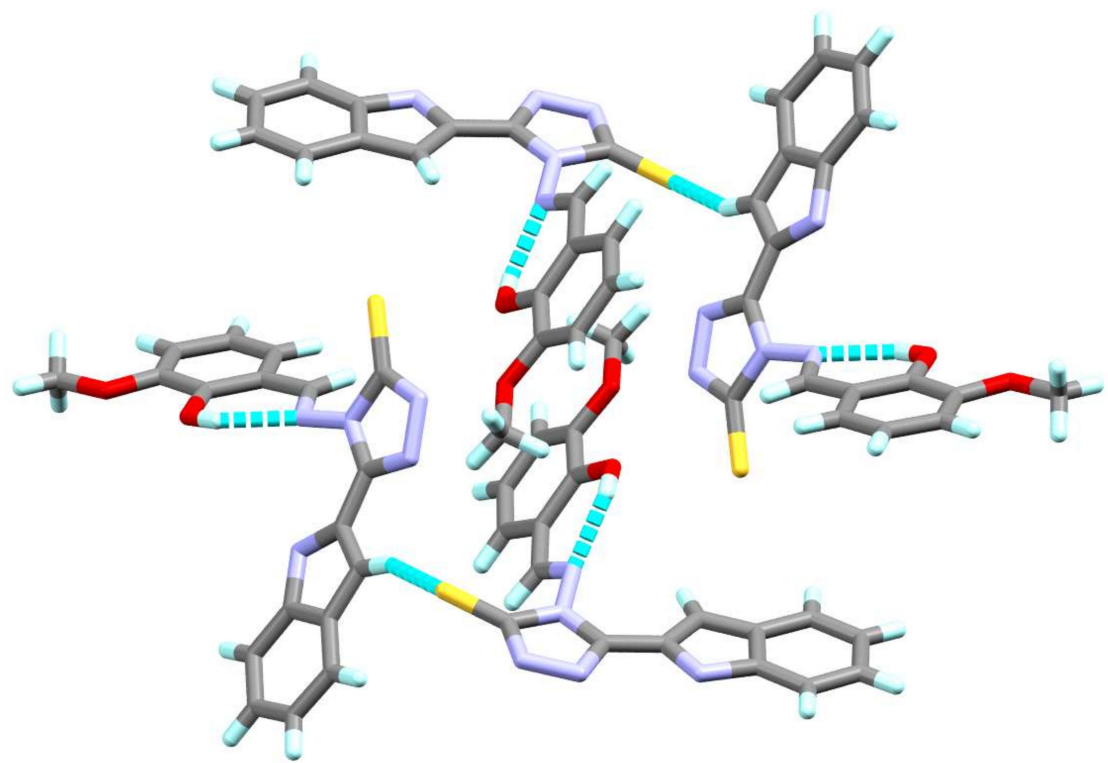

Figure 4. The molecular packing of the synthesized compound 6. Intra and intermolecular hydrogen bonds are indicated as dashed lines. 
The structure showed two intramolecular hydrogen bonds of the type $\mathrm{O}-\mathrm{H} \ldots \mathrm{N}$ between the hydroxyl group as a hydrogen bond donor, the adjacent Schiff base nitrogen atom as a hydrogen bond acceptor, and donor-acceptor distances in the range of 2.659(6)-2.664(6) A (Table 3). In addition, each of the two units in the crystal are connected by weak C11-H11 ... S2 hydrogen bonds with a donor-acceptor distance of 3.391(5) $\AA$, leading to the hydrogen-bonded dimeric units shown in Figure 4 .

Table 3. Hydrogen bond parameters for 6 .

\begin{tabular}{ccccc}
\hline D-H . . A & D-H & H . . A & D . . A & D-H . . A \\
\hline O1-H1 . . N1 & 0.84 & 1.94 & $2.659(6)$ & 143 \\
O3-H3A . . N6 & 0.84 & 1.95 & $2.664(6)$ & 143 \\
C11-H11 . . S2 & 0.95 & 2.51 & $3.391(5)$ & 154 \\
\hline
\end{tabular}

\section{Materials and Methods}

Melting points are determined using a melting-point apparatus (SMP10) in open capillaries and are uncorrected. The progress of the reactions was monitored by thin layer chromatography (Merck). Detections were achieved by UV light illumination. For flash chromatography, commercial silica was used. Nuclear magnetic resonance $\left({ }^{1} \mathrm{H}-\mathrm{NMR},{ }^{13} \mathrm{C}-\mathrm{NMR}\right.$, and 2D NMR) spectra were determined in DMSO- $d_{6}$ and were recorded on Bruker AC 300/500 spectrometers using TMS as an internal standard. Chemical shifts are termed in $\delta$ (ppm) and coupling constants are described in Hz. The assignment of exchangeable $\mathrm{OH}$ and $\mathrm{NH}$ was confirmed by $\mathrm{D}_{2} \mathrm{O}$. CHNS-microanalysis was done using a Flash EA-1112 instrument. The HREI mass spectra were detected using a Finnigan MAT 95XP. The FAB-MS was done using Jeol JMS HX110. The IR were detected using a Bruker Alpha ATR-FTIR.

\subsection{Procedure for $\mathbf{2}-\mathbf{6}$}

The appropriate aldehyde ( $1.1 \mathrm{mmol})$ and 4-amino-5-(1H-indol-2-yl)-2,4-dihydro-3H-1,2,4-triazole3-thione $1(1.0 \mathrm{mmol})$ were refluxed in glacial acetic acid $(5.0 \mathrm{~mL})$ for $3 \mathrm{~h}$, then cooled, crystals either appear during cooling then filtered and recrystallized from ethanol or the mixture was poured into cold water; the formed ppt was filtered, dried, and purified by silica-column chromatography using $\mathrm{EA} / \mathrm{H}(1: 1)$ as an eluent.

4-(Benzylideneamino)-5-(1H-indol-2-yl)-2H-1,2,4-triazole-3(4H)-thione 2. Yield 65\%; m.p. 221 to $222{ }^{\circ} \mathrm{C},{ }^{1} \mathrm{H}-\mathrm{NMR}$ (DMSO- $\left.d_{6}, 500 \mathrm{MHz}\right) \delta 7.03(\mathrm{dd}, 1 \mathrm{H}, J$ 8.0, J $7.5 \mathrm{~Hz}), 7.08(\mathrm{~d}, 1 \mathrm{H}, J 1.0 \mathrm{~Hz}), 7.20(\mathrm{dd}, 1 \mathrm{H}, J$ 7.5, J 8.2 Hz), $7.45(\mathrm{~d}, 1 \mathrm{H}, J 8.2 \mathrm{~Hz}), 7.61$ to $7.70(\mathrm{~m}, 4 \mathrm{H}), 8.02(\mathrm{~d}, 2 \mathrm{H}, J 7.3 \mathrm{~Hz}), 9.73(\mathrm{~s}, 1 \mathrm{H}, \mathrm{CH}=\mathrm{N}), 11.89$ (br. s, $1 \mathrm{H}$, $\left.\mathrm{NH}_{\text {Indole }}\right), 14.28$ (br. s, $\left.\mathrm{H}, \mathrm{NH}_{\text {Triazole }}\right) ;{ }^{13} \mathrm{C}-\mathrm{NMR}$ (DMSO- $\left.d_{6}, 125 \mathrm{MHz}\right) \delta 105.30,111.88,119.87,121.25$, 122.34, 123.69, 127.26, 128.87, 129.30, 131.90, 132.95, 136.93, 143.41, 162.09, 167.12; IR (cm $\left.{ }^{-1}\right): 1601,2923$, 3223 , 3423; elemental analysis calculation for $\mathrm{C}_{17} \mathrm{H}_{13} \mathrm{~N}_{5} \mathrm{~S}: \mathrm{C}, 63.93 ; \mathrm{H}, 4.10 ; \mathrm{N}, 21.93 ; \mathrm{S}, 10.04$ found: $\mathrm{C}$, 63.63; H, 3.95; N, 21.99; S, 10.15 .

(E)-4-(4-Flourobenzylideneamino)-5-(1H-indol-2-yl)-2H-1,2,4-triazole-3(4H)-thione 3. Yield: 71\%; m.p. 227 to $228^{\circ} \mathrm{C},{ }^{1} \mathrm{H}-\mathrm{NMR}$ (DMSO- $\left.d_{6}, 3400 \mathrm{MHz}\right) \delta 7.03$ (ddd, $1 \mathrm{H}, J$ 8.0, J 7.5, J $\left.0.9 \mathrm{~Hz}\right), 7.09$ (d, $1 \mathrm{H}, J 1.03 \mathrm{~Hz}$ ), $7.22(\mathrm{ddd}, 1 \mathrm{H}, J$ 7.5, J 8.2, J $1.1 \mathrm{~Hz}), 7.46$ to $7.50(3,3 \mathrm{H}), 7.63(\mathrm{~d}, 1 \mathrm{H}, J 8.0 \mathrm{~Hz}), 8.11$ to $8.15(\mathrm{~m}, 2 \mathrm{H})$, $9.74(\mathrm{~s}, 1 \mathrm{H}, \mathrm{CH}=\mathrm{N}), 11.90$ (br. s, $\left.1 \mathrm{H}, \mathrm{NH}_{\text {Indole }}\right), 14.30$ (br. s, $\mathrm{H}, \mathrm{NH}_{\text {Triazole }}$ ); ${ }^{13} \mathrm{C}-\mathrm{NMR}$ (DMSO- $d_{6}, 100$ MHz) $\delta 105.36,111.93,116.49,116.71,119.93,121.29,122.33,123.76,127.29,128.58,131.50,131.59,136.97$, 143.43, 162.09, 163.66, 166.15; IR ( $\left.\mathrm{cm}^{-1}\right)$ : 1603, 2927, 3099, 3467; elemental analysis calculation for $\mathrm{C}_{17} \mathrm{H}_{12} \mathrm{FN}_{5} \mathrm{~S}: \mathrm{C}, 60.52 ; \mathrm{H}, 3.59 ; \mathrm{F}, 5.63 ; \mathrm{N}, 20.76 ; \mathrm{S}, 9.50$ found: $\mathrm{C}, 60.26 ; \mathrm{H}, 3.66 ; \mathrm{F}, 5.71 ; \mathrm{N}, 21.01 ; \mathrm{S}, 9.55$.

(E)-4-((3-Bromobenzylidene)amino)-5-(1H-indol-2-yl)-2,4-dihydro-3H-1,2,4-triazole-3-thione 4. Yield: 49\%; m.p. 236 to $237^{\circ} \mathrm{C},{ }^{1} \mathrm{H}-\mathrm{NMR}$ (DMSO- $\left.d_{6}, 400 \mathrm{MHz}\right) \delta 7.04$ to $7.08(\mathrm{~m}, 2 \mathrm{H}), 7.22(\mathrm{dd}, 1 \mathrm{H}, J$ J.5, J 8.2 Hz), $7.47(\mathrm{~d}, 1 \mathrm{H}, J$ 8 $8.2 \mathrm{~Hz}), 7.58-7.67(\mathrm{~m}, 2 \mathrm{H}), 7.89(\mathrm{~d}, 1 \mathrm{H}, J 8.0 \mathrm{~Hz}), 8.06(\mathrm{~d}, 1 \mathrm{H}, J$ J.8 Hz), $8.19(\mathrm{~s}, 1 \mathrm{H}), 9.80$ (s, $1 \mathrm{H}, \mathrm{CH}=\mathrm{N}$ ), 11.91 (br. s, $1 \mathrm{H}, \mathrm{NH}_{\text {Indol }}$ ), 14.34 (br. s, $\mathrm{H}, \mathrm{NH}_{\text {Triazol }}$ ); ${ }^{13} \mathrm{C}-\mathrm{NMR}$ (DMSO- $\left.d_{6}, 100 \mathrm{MHz}\right) \delta$ 
$105.93,112.43,120.45,121.81,122.71,122.96,124.29,127.76,128.18,131.80,132.03,134.75,136.01,137.48$, $143.98,162.60,166.02 ; \mathrm{IR}\left(\mathrm{cm}^{-1}\right)$ : $1588,2928,3087,3442$; elemental analysis calculation for $\mathrm{C}_{17} \mathrm{H}_{12} \mathrm{BrN}_{5} \mathrm{~S}$ : C, 51.27; H, 3.04; Br, 20.06; N, 17.58; S, 8.05. Found: C, 51.08; H, 3.22; Br, 20.13; N, 17.68; S, 8.11.

(E)-5-(1H-Indol-2-yl)-4-((4-methylbenzylidene)amino)-2,4-dihydro-3H-1,2,4-triazole-3-thione 5. Yield: 45\%; m.p. 225 to $226^{\circ} \mathrm{C}^{1}{ }^{1} \mathrm{H}-\mathrm{NMR}$ (DMSO- $\left.d_{6}, 300 \mathrm{MHz}\right) \delta 2.42\left(\mathrm{~s}, 3 \mathrm{H}, \mathrm{CH}_{3}\right.$ ), 6.99 to 7.05 (m, $2 \mathrm{H}$ ), 7.19 (dd, 1H, J 7.5, J 8.2 Hz), 7.42 to $7.45(\mathrm{~m}, 3 \mathrm{H}), 7.61(\mathrm{~d}, 1 \mathrm{H}, \mathrm{J} 7.9 \mathrm{~Hz}), 7.91(\mathrm{~d}, 2 \mathrm{H}, J 7.9 \mathrm{~Hz}), 9.62(\mathrm{~s}, 1 \mathrm{H}$, $\mathrm{CH}=\mathrm{N}$ ), 11.88 (br. s, $1 \mathrm{H}, \mathrm{NH}_{\text {Indole }}$ ), 14.25 (br. s, $\mathrm{H}, \mathrm{NH}_{\text {Triazole }}$ ); ${ }^{13} \mathrm{C}-\mathrm{NMR}$ (DMSO- $d_{6}, 100 \mathrm{MHz}$ ) $\delta 21.8$, 105.8, 112.4, 120.4, 121.7, 123.0, 124.2, 127.8, 129.4, 129.8, 130.4, 137.5, 143.9, 167.6; IR (cm $\left.{ }^{-1}\right): 1602,2924$, 3225,3420 elemental analysis calculation for $\mathrm{C}_{18} \mathrm{H}_{15} \mathrm{~N}_{5} \mathrm{~S}: \mathrm{C}, 64.84 ; \mathrm{H}, 4.53 ; \mathrm{N}, 21.01 ; \mathrm{S}, 9.62$. Found: $\mathrm{C}$, $64.96 ; \mathrm{H}, 4.67 ; \mathrm{N}, 20.95 ; \mathrm{S}, 9.39$.

(E)-4-((2-Hydroxy-3-methoxybenzylidene)amino)-5-(1H-indol-2-yl)-2,4-dihydro-3H-1,2,4-triazole-3-thione 6. Yield: $61 \%$; m.p. 230 ot $231^{\circ} \mathrm{C},{ }^{1} \mathrm{H}-\mathrm{NMR}$ (DMSO- $\left.d_{6}, 500 \mathrm{MHz}\right) \delta 3.87\left(\mathrm{~s}, 3 \mathrm{H}, \mathrm{CH}_{3}\right), 7.03$ to $7.07(\mathrm{~m}, 3$ $\mathrm{H}), 7.17$ to $7.23(\mathrm{~m}, 2 \mathrm{H}), 7.45(\mathrm{~d}, 1 \mathrm{H}, J 8.4 \mathrm{~Hz}), 7.59$ to $7.63(\mathrm{~m}, 2 \mathrm{H}), 9.88(\mathrm{~s}, 1 \mathrm{H}), 9.99(\mathrm{~s}, 1 \mathrm{H}, \mathrm{CH}=\mathrm{N})$, 11.87 (br. s, $\left.1 \mathrm{H}, \mathrm{NH}_{\text {Indole }}\right), 14.23$ (br. s, $\left.\mathrm{H}, \mathrm{NH}_{\text {Triazole }}\right) ;{ }^{13} \mathrm{C}-\mathrm{NMR}$ (DMSO- $\left.d_{6}, 75 \mathrm{MHz}\right) \delta 56.14\left(\mathrm{CH}_{3}\right)$, 105.23, 111.934, 115.78, 1168.52, 118.60, 119.69, 119.91, 121.27, 122.54, 123.69, 127.30, 136.93, 143.49, 148.37, 148.47, 162.07, 163.24; IR ( $\left.\mathrm{cm}^{-1}\right)$ : 1608, 2915, 3227, 3418; elemental analysis calculation for $\mathrm{C}_{18} \mathrm{H}_{15} \mathrm{~N}_{5} \mathrm{O}_{2} \mathrm{~S}: \mathrm{C}, 59.17 ; \mathrm{H}, 4.14 ; \mathrm{N}, 8.76 ; \mathrm{S}, 8.77$ found: $\mathrm{C}, 59.43 ; \mathrm{H}, 4.22 ; \mathrm{N}, 18.97 ; \mathrm{S}, 8.75$.

\subsection{General Procedure for the Synthesis of Indolo-Triazolo-Pyridazinethiones 7-16}

To a mixture of indolyltriazolethione $\mathbf{1}(1.0 \mathrm{mmol})$ in ethanol $(5.0 \mathrm{~mL})$, the appropriate aldehyde $(1.1 \mathrm{mmol})$ was added followed by the addition of 5 drops of concentrated $\mathrm{HCl}$, and the mixture was refluxed for 1 to $2 \mathrm{~h}$ until a precipitate was formed. The solid product was cooled, filtered, dried, and recrystallized from ethanol.

5,6-Dihydro-14H-indolo[2,3-d]-6-phenyl-[1,2,4-triazolo][4,3-b]pyridazine-3(2H)thione 7. Yield: 89\%; m.p. $>300{ }^{\circ} \mathrm{C} ;{ }^{1} \mathrm{H}-\mathrm{NMR}$ (DMSO-d $\left.6,500 \mathrm{MHz}\right) \delta 5.92\left(\mathrm{~d}, 1 \mathrm{H}, J 5.7 \mathrm{~Hz}, \mathrm{H}-6_{\text {Pyridazine }}\right), 7.01$ (d, $1 \mathrm{H}, J 5.7 \mathrm{~Hz}$, $\mathrm{HN}_{\text {Pyridazine, }} \mathrm{D}_{2} \mathrm{O}$ exchangeable), $7.07(\mathrm{dd}, 1 \mathrm{H}, J \approx 7.5 \mathrm{~Hz}), 7.22$ to $7.29(\mathrm{~m}, 4 \mathrm{H}), 7.41-7.48(\mathrm{~m}, 4 \mathrm{H}), 12.24$ (br. s, $1 \mathrm{H}, \mathrm{NH}_{\text {Indole }}, \mathrm{D}_{2} \mathrm{O}$ exchangeable), 13.64 (br. s, $1 \mathrm{H}, \mathrm{NH}_{\text {Triazole }}, \mathrm{D}_{2} \mathrm{O}$ exchangeable); ${ }^{13} \mathrm{C}-\mathrm{NMR}$ (DMSO- $\left.d_{6}, 125 \mathrm{MHz}\right) \delta 55.51$ (C-6Pyridazine), 112.43, 116.79, 119.27, 119.74, 120.40, 124.08, 124.17, 127.065, $127.49,128.25,137.80,139.91,141.83,164.21$; IR $\left(\mathrm{cm}^{-1}\right): 1628,2897,2985,3067,3162,3222$; HRMS (EI) calculated for $\mathrm{C}_{17} \mathrm{H}_{13} \mathrm{~N}_{5} \mathrm{~S}\left(\mathrm{M}^{+}\right)$: 319.0892. Found: 319.0902 .

5,6-Dihydro-6-(4-flourophenyl)-14H-indolo[2,3-d]-[1,2,4-triazolo][4,3-b]pyridazine-3(2H)thione 8. Yield: 80\%; m.p. $>300{ }^{\circ} \mathrm{C} ;{ }^{1} \mathrm{H}-\mathrm{NMR}$ (DMSO- $\left.d_{6}, 300 \mathrm{MHz}\right) \delta 5.93(\mathrm{~d}, 1 \mathrm{H}, J 5.4 \mathrm{~Hz}, \mathrm{H}-6$ Pyridazine) $) 7.04(\mathrm{~d}, 1 \mathrm{H}, J$

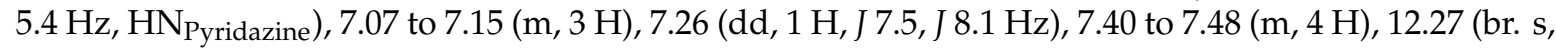
$\left.1 \mathrm{H}, \mathrm{NH}_{\text {Indole }}\right), 13.66$ (br. s, $\left.1 \mathrm{H}, \mathrm{NH}_{\text {Triazole }}\right) ;{ }^{13} \mathrm{C}-\mathrm{NMR}$ (DMSO-d 6 , $\left.75 \mathrm{MHz}\right) \delta 54.81$ (C-6Pyridazin $), 112.51$, 114.93, 115.21, 116.61, 119.34, 119.72, 120.54, 124.14, 124.19, 129.08, 129.19, 136.12, 137.83, 141.83, 163.14 $\left(\mathrm{C}_{\mathrm{Ph}}\right), 164.32$; IR $\left(\mathrm{cm}^{-1}\right): 1604,1632,2907,2960,3068,3265$; HRMS (EI) calculated for $\mathrm{C}_{17} \mathrm{H}_{12} \mathrm{~N}_{5} \mathrm{SF}\left(\mathrm{M}^{+}\right)$: 337.0797. Found: 337.0781 .

6-(3-Bromophenyl)-5,6-dihydro-14H-indolo[2,3-d]-[1,2,4-triazolo][4,3-b]pyridazine-3(2H)thione 9. Yield: 73\%; m.p. $>300{ }^{\circ} \mathrm{C} ;{ }^{1} \mathrm{H}-\mathrm{NMR}$ (DMSO- $\left.d_{6}, 300 \mathrm{MHz}\right) \delta 5.99$ (d, $\left.1 \mathrm{H}, J 5.1 \mathrm{~Hz}, \mathrm{H}-6_{\text {Pyridazine }}\right), 7.11$ to 7.35 (m, $2 \mathrm{H}), 7.43(\mathrm{~d}, 1 \mathrm{H}, J 8.1 \mathrm{~Hz}), 7.48(\mathrm{~d}, 1 \mathrm{H}, J 8.1 \mathrm{~Hz}), 7.59(\mathrm{~d}, 1 \mathrm{H}, J 7.8 \mathrm{~Hz}), 7.69(\mathrm{~s}, 1 \mathrm{H}),$,12.30 (br. s, $1 \mathrm{H}, \mathrm{NH}_{\text {Indole }}$ ), 13.68 (br. s, $1 \mathrm{H}, \mathrm{NH}_{\text {Triazole }}{ }^{13} \mathrm{C}-\mathrm{NMR}$ (DMSO-d $\left.6,75 \mathrm{MHz}\right) \delta 55.10$ (C-6yridazin $), 113.05$, 116.67, 119.80, 120.18, 121.17, 122.18, 124.65, 124.77, 126.48, 129.08, 130.19, 130.83, 130.98, 138.27, 142.27, 143.34, 164.93; IR $\left(\mathrm{cm}^{-1}\right): 1627,2906,3075,3202,3263$; HRMS (EI) calculated for $\mathrm{C}_{17} \mathrm{H}_{12} \mathrm{~N}_{5} \mathrm{SBr}\left(\mathrm{M}^{+}\right)$: 396.9997. Found: 397.0000 . 
5,6-Dihydro-14H-indolo[2,3-d]-6-p-tolyl-5,6-[1,2,4-triazolo][4,3-b]pyridazine-3(2H)thione 10. Yield: 91\%; m.p. $>300{ }^{\circ} \mathrm{C} ;{ }^{1} \mathrm{H}-\mathrm{NMR}$ (DMSO-d $\left.6,300 \mathrm{MHz}\right) \delta 2.23\left(\mathrm{~s}, 3 \mathrm{H}, \mathrm{CH}_{3}\right), 5.84$ (d, $1 \mathrm{H}, \mathrm{J} 5.8 \mathrm{~Hz}, \mathrm{H}-6_{\text {Pyridazine }}$ ), $6.93\left(\mathrm{~d}, 1 \mathrm{H}, J 5.8 \mathrm{~Hz}, \mathrm{HN}_{\text {Pyridazine }}\right), 7.04-7.28$ (m, $\left.6 \mathrm{H}\right), 7.39$ (d, $\left.1 \mathrm{H}, J 8.0 \mathrm{~Hz}\right), 7.46$ (d, 1 H, J 8.2 Hz), 12.22 (br. s, $\left.1 \mathrm{H}, \mathrm{NH}_{\text {Indole }}\right), 13.62$ (br. s, $\left.1 \mathrm{H}, \mathrm{NH}_{\text {Triazole }}\right) ;{ }^{13} \mathrm{C}-\mathrm{NMR}\left(\mathrm{DMSO}-d_{6}, 75 \mathrm{MHz}\right) \delta 20.62\left(\mathrm{CH}_{3}\right)$, 55.44 (C-6 Pyridazin), 112.45, 116.93, 119.34, 119.77, 120.39, 124.09, 124.18, 127.15, 128.84, 136.76, 136.85, 137.83, 141.89, 164.20; IR ( $\left.\mathrm{cm}^{-1}\right)$ : 1622, 3192, 3322; HRMS (EI) calculated for $\mathrm{C}_{18} \mathrm{H}_{15} \mathrm{~N}_{5} \mathrm{~S}\left(\mathrm{M}^{+}\right)$: 333.1048 . Found: 333.1072 .

5,6-Dihydro-14H-indolo[2,3-d]-6-(2,3,4-trimethoxyphenyl)-[1,2,4-triazolo][4,3-b]pyridazine-3(2H)thione 11. Yield: 93\%; m.p. 266 to $267{ }^{\circ} \mathrm{C}$; ${ }^{1} \mathrm{H}-\mathrm{NMR}$ (DMSO- $\left.d_{6}, 300 \mathrm{MHz}\right) \delta 3.72,3.79,3.96\left(3 \mathrm{~s}, 9 \mathrm{H}, 3 \mathrm{OCH}_{3}\right)$, $5.99(\mathrm{~d}, 1 \mathrm{H}, J 7.1 \mathrm{~Hz}, \mathrm{H}-6$ Pyridazine), 6.40 (d, $1 \mathrm{H}, \mathrm{J} 8.8 \mathrm{~Hz}), 6.63(\mathrm{~d}, 1 \mathrm{H}, J 8.8 \mathrm{~Hz}), 6.75$ (d, $1 \mathrm{H}, J 7.1 \mathrm{~Hz}$,

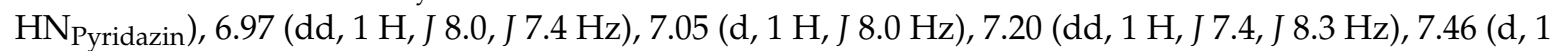
H, J $8.3 \mathrm{~Hz}$ ), 12.26 (br. s, $\left.1 \mathrm{H}, \mathrm{NH}_{\text {Indole }}\right), 13.63$ (br. s, $1 \mathrm{H}, \mathrm{NH}_{\text {Triazole }}$ ); ${ }^{13} \mathrm{C}-\mathrm{NMR}$ (DMSO- $\left.d_{6}, 100 \mathrm{MHz}\right) \delta$ 51.15, 55.70, 60.36, 61.57 (C-6Pyridazin, $3 \mathrm{OCH}_{3}$ ), 107.41, 112.44, 115.44, 119.51, 120.18, 120.27, 122.96, 123.54, 124.06, 124.67, 137.94, 141.77, 141.89, 151.63, 153.42, 163.64; IR (cm $\left.{ }^{-1}\right):$ 1623, 3001, 3175; HRMS (EI) calculated for $\mathrm{C}_{20} \mathrm{H}_{19} \mathrm{~N}_{5} \mathrm{O}_{3} \mathrm{~S}\left(\mathrm{M}^{+}\right)$: 409.1209. Found: 409.1213 .

6-(4-Chlorophenyl)-5,6-dihydro-14H-indolo[2,3-d]-[1,2,4-triazolo][4,3-b]pyridazine-3(2H)thione 12. Yield: 68\%; m.p. $>300{ }^{\circ} \mathrm{C} ;{ }^{1} \mathrm{H}-\mathrm{NMR}$ (DMSO- $\left.d_{6}, 300 \mathrm{MHz}\right) \delta 5.96$ (d, $1 \mathrm{H}, J 5.4 \mathrm{~Hz}, \mathrm{H}-6$ Pyridazine), 7.08 to 7.14 $(\mathrm{m}, 2 \mathrm{H}), 7.27(\mathrm{dd}, 1 \mathrm{H}, J$ 7.5, J $8.1 \mathrm{~Hz}), 7.34(\mathrm{~d}, 2 \mathrm{H}, \mathrm{J} 8.4 \mathrm{H}), 7.41(\mathrm{~d}, 2 \mathrm{H}, J 8.4 \mathrm{H}), 7.48(\mathrm{~d}, 1 \mathrm{H}, J 8.1 \mathrm{~Hz})$, 7.53 (d, 1 H, J $7.8 \mathrm{~Hz}$ ), 12.28 (br. s, $\left.1 \mathrm{H}, \mathrm{NH}_{\text {Indole }}\right), 13.67$ (br. s, $1 \mathrm{H}, \mathrm{NH}_{\text {Triazole }}$ ); ${ }^{13} \mathrm{C}-\mathrm{NMR}\left(\mathrm{DMSO}-d_{6}\right.$, $75 \mathrm{MHz}$ ) $\delta 54.69$ (C-6 Pyridazine), 112.53, 116.32, 119.36, 119.72, 120.60, 124.16, 124.23, 128.28, 128.94, 132.17, 137.82, 139.07, 141.80, 164.36; IR ( $\left.\mathrm{cm}^{-1}\right)$ : 1635, 2915, 3075, 3160, 3268; HRMS (EI) calculated for $\mathrm{C}_{17} \mathrm{H}_{12} \mathrm{~N}_{5} \mathrm{SCl}\left(\mathrm{M}^{+}\right)$: 353.0502. Found: 353.0500 .

6-(4-Bromophenyl)-5,6-dihydro-14H-indolo[2,3-d]-[1,2,4-triazolo][4,3-b]pyridazine-3(2H)thione 13. Yield 71\%; m.p. $>300{ }^{\circ} \mathrm{C} ;{ }^{1} \mathrm{H}-\mathrm{NMR}$ (DMSO-d $\left.6,300 \mathrm{MHz}\right) \delta 5.95$ (d, $1 \mathrm{H}, \mathrm{J} 5.1 \mathrm{~Hz}, \mathrm{H}-6$ Pyridazine), 7.10 to 7.55 $(\mathrm{m}, 9 \mathrm{H}), 12.29$ (br. s, $\left.1 \mathrm{H}, \mathrm{NH}_{\text {Indole }}\right), 13.68$ (br. s, $\left.1 \mathrm{H}, \mathrm{NH}_{\text {Triazole }}\right) ;{ }^{13} \mathrm{C}-\mathrm{NMR}\left(\mathrm{DMSO}-d_{6}, 100 \mathrm{MHz}\right) \delta$ 54.75 (C-6 $\left.6_{\text {Pyridazine }}\right), 112.55,116.27,119.39,119.74,120.63,124.19,124.26,129.33,131.22,137.85,139.54$, 141.82, 164.38; IR (cm $\left.{ }^{-1}\right)$ : 1629, 2907, 3071, 3202, 3268; HRMS (EI) calculated for $\mathrm{C}_{17} \mathrm{H}_{12} \mathrm{~N}_{5} \mathrm{SBr}\left(\mathrm{M}^{+}\right)$: 396.9997. Found: 396.9964 .

5,6-Dihydro-6-(3,4-dihydroxyphenyl)-14H-indolo[2,3-d]-[1,2,4-triazolo][4,3-b]pyridazine-3(2H)thione 14. Yield 74\%; m.p. $>300{ }^{\circ} \mathrm{C}$; ${ }^{1} \mathrm{H}-\mathrm{NMR}$ (DMSO-d $\left.6,300 \mathrm{MHz}\right) \delta 5.66$ (d, $1 \mathrm{H}, \mathrm{J} 6.3 \mathrm{~Hz}, \mathrm{H}-6_{\text {Pyridazine }}$ ), 6.61 to $6.74(\mathrm{~m}, 4 \mathrm{H}), 7.03(\mathrm{dd}, 1 \mathrm{H}, \mathrm{J} 8.1 \mathrm{~J} 6.9 \mathrm{~Hz}), 7.20-7.25(\mathrm{~m}, 2 \mathrm{H}), 7.45(\mathrm{~d}, 1 \mathrm{H}, J 8.1 \mathrm{~Hz}), 8.78(\mathrm{~s}, 1 \mathrm{H}$, $\mathrm{OH}), 8.87$ (s, $1 \mathrm{H}, \mathrm{OH}), 12.18$ (br. s, $1 \mathrm{H}, \mathrm{NH}_{\text {Indole }}$ ), 13.60 (br. s, $1 \mathrm{H}, \mathrm{NH}_{\text {Triazole }}$ ); ${ }^{13} \mathrm{C}-\mathrm{NMR}\left(\mathrm{DMSO}-d_{6}\right.$, $100 \mathrm{MHz}) \delta 55.89$ (C-6 Pyridazine), 112.38, 115.16, 115.23, 117.08, 118.60, 119.40, 119.90, 120.23, 124.01, 124.16, 130.42, 137.87, 141.86, 144.97, 145.01, 163.90; IR $\left(\mathrm{cm}^{-1}\right):$ 1634, 2908, 3071, 3313; HRMS (EI) calculated for $\mathrm{C}_{17} \mathrm{H}_{13} \mathrm{O}_{2} \mathrm{~N}_{5} \mathrm{~S}\left(\mathrm{M}^{+}\right)$: 351.0790. Found: 351.0765 .

5,6-Dihydro-6-(4-hydroxy-3-methoxyphenyl)-14H-indolo[2,3-d]-[1,2,4-triazolo][4,3-b]pyridazine-3(2H)thione 15. Yield $87 \%$; m.p. 300 to $301{ }^{\circ} \mathrm{C} ;{ }^{1} \mathrm{H}-\mathrm{NMR}$ (DMSO- $\left.d_{6}, 300 \mathrm{MHz}\right) \delta 3.70\left(\mathrm{~s}, 3 \mathrm{H}, \mathrm{OCH}_{3}\right), 5.75$ (d, $1 \mathrm{H}, J$ $\left.6.0 \mathrm{~Hz}, \mathrm{H}-6_{\text {Pyridazine }}\right), 6.57$ to $6.64(\mathrm{~m}, 2 \mathrm{H}), 6.86(\mathrm{~d}, 1 \mathrm{H}, J 6.0 \mathrm{~Hz}), 7.07(\mathrm{dd}, 1 \mathrm{H}, J$ 7.8, J 7.2 Hz), 7.21 to 7.28 (m, $2 \mathrm{H}), 7.38$ (d, $1 \mathrm{H}, J 7.8 \mathrm{~Hz}), 7.45$ (d, $1 \mathrm{H}, \mathrm{J} 8.1 \mathrm{~Hz}), 8.91$ (s, $1 \mathrm{H}, \mathrm{OH}), 12.19$ (br. s, 1H, NH Indole), 13.62 (br. s, $\left.1 \mathrm{H}, \mathrm{NH}_{\text {Triazole }}\right) ;{ }^{13} \mathrm{C}-\mathrm{NMR}\left(\mathrm{DMSO}-d_{6}, 75 \mathrm{MHz}\right) \delta 55.53,55.64\left(\mathrm{C}-6_{\text {Pyridazine, }}, \mathrm{OCH}_{3}\right), 111.49$, $112.43,115.15,117.49,119.25,119.77,119.82,120.36,124.04,124.32,130.40,137.77,141.98,145.89,147.37$, 164.25; IR (cm $\left.{ }^{-1}\right): 1633,2917,3074,3167,3279$, 3401; HRMS (EI) calculated for $\mathrm{C}_{18} \mathrm{H}_{15} \mathrm{~N}_{5} \mathrm{O}_{2} \mathrm{~S}\left(\mathrm{M}^{+}\right)$: 365.0946. Found: 365.0980 .

5,6-Dihydro-6-(2-hydroxy-3-methoxyphenyl)-14H-indolo[2,3-d]-[1,2,4-triazolo][4,3-b]pyridazine-3(2H)thione 16. Yield 89\%; m.p. 200 to $201{ }^{\circ} \mathrm{C} ;{ }^{1} \mathrm{H}-\mathrm{NMR}$ (DMSO- $\left.d_{6}, 500 \mathrm{MHz}\right) \delta 3.82\left(\mathrm{~s}, 3 \mathrm{H}, \mathrm{OCH}_{3}\right), 6.15$ (d, $1 \mathrm{H}, J$ 
$\left.6.5 \mathrm{~Hz}, \mathrm{H}-6_{\text {Pyridazine }}\right), 6.27$ (d, $\left.1 \mathrm{H}, J 7.6 \mathrm{~Hz}\right), 6.60(\mathrm{dd}, 1 \mathrm{H}, J$ 7.9, J $8.0 \mathrm{~Hz}), 6.87$ to 6.91 (m, $\left.2 \mathrm{H}\right), 6.96$ $(\mathrm{dd}, 1 \mathrm{H}, J$ J 8.0, J $7.3 \mathrm{~Hz}), 7.10(\mathrm{~d}, 1 \mathrm{H}, J 8.0 \mathrm{~Hz}), 7.21(\mathrm{dd}, 1 \mathrm{H}, J$ 7.3, J $8.3 \mathrm{~Hz}), 7.45(\mathrm{~d}, 1 \mathrm{H}, J 8.3 \mathrm{~Hz})$, 9.27 (s, $1 \mathrm{H}, \mathrm{OH}), 12.24$ (br. s, $\left.1 \mathrm{H}, \mathrm{NH}_{\text {Indole }}\right), 13.68$ (br. s, $1 \mathrm{H}, \mathrm{NH}_{\text {Triazole }}$ ); ${ }^{13} \mathrm{C}-\mathrm{NMR}$ (DMSO- $d_{6}, 125$ MHz) $\delta 51.15$ (C-6 Pyridazin $), 55.84\left(\mathrm{OCH}_{3}\right), 111.51,112.32,115.64,118.75,119.54,119.75,119.87,120.09$, 123.71, 124.01, 125.72, 137.91, 141.15, 143.94, 147.54, 162.67; IR ( $\left.\mathrm{cm}^{-1}\right): 1628,2920,3074,3167,3280,3406$; HRMS (FAB +ve) calculated for $\mathrm{C}_{18} \mathrm{H}_{16} \mathrm{~N}_{5} \mathrm{O}_{2} \mathrm{~S}\left(\mathrm{M}^{+}\right)$: 366.1025. Found: 366.091030 .

\subsection{General Procedure for the Alkylation with Di(bromomethyl)quinoxaline 19-25}

The appropriate indolo-triazolo-pyridazinethiones $(2.0 \mathrm{mmol})$ and $\mathrm{K}_{2} \mathrm{CO}_{3}(2.2 \mathrm{mmol})$ were stirred in acetone $(10 \mathrm{~mL})$ for $1 \mathrm{~h}$; then, di(bromomethyl)quinoxaline $(1.1 \mathrm{mmol})$ was added and stirring was continued overnight. The solvent was removed under vacuum, water was added, and the solid was obtained by filtration, dried, and recrystallized from dimethyl formamide (DMF).

2,3-Bis((5,6-Dihydro-14H-indolo[2,3-d]-6-phenyl-[1,2,4-triazolo][4,3-b]pyridazin-3-ylsulfanyl)methyl)quinoxaline 19. Yield: 83\%; m.p. 291 to $292{ }^{\circ} \mathrm{C}$; ${ }^{1} \mathrm{H}-\mathrm{NMR}$ (DMSO- $\left.d_{6}, 300 \mathrm{MHz}\right) \delta 5.04\left(\mathrm{~s}, 4 \mathrm{H}, 2 \mathrm{SCH}_{2}\right), 5.91(\mathrm{~d}, 2 \mathrm{H}, J$ $6.3 \mathrm{~Hz}, 2 \mathrm{H}-6$ Pyridazine), $7.40(\mathrm{dd}, 2 \mathrm{H}, J \approx 7.5 \mathrm{~Hz}), 7.20$ to $7.36(\mathrm{~m}, 16 \mathrm{H}), 7.47(\mathrm{~d}, 2 \mathrm{H}, J 8.1 \mathrm{~Hz}), 7.80$ to $7.83(\mathrm{~m}, 2 \mathrm{H}), 8.00-8.03$ (m, $2 \mathrm{H}), 12.34$ (br. s, $2 \mathrm{H}, 2 \mathrm{NH}_{\text {Indole }}$ ); ${ }^{13} \mathrm{C}-\mathrm{NMR}$ (DMSO- $\left.d_{6}, 75 \mathrm{MHz}\right) \delta 35.86(2$ $\left.\mathrm{SCH}_{2}\right), 56.54$ (2 C-6yridazine), 112.85, 114.53, 119.94, 120.71, 121.55, 123.92, 124.96, 127.73, 128.13, 128.71, 128.83, 130.74, 137.91, 140.29, 140.53, 146.53, 149.00, 151.10; IR $\left(\mathrm{cm}^{-1}\right)$ : 1611, 2926, 3143; elemental analysis calculated for $\mathrm{C}_{44} \mathrm{H}_{32} \mathrm{~N}_{12} \mathrm{~S}_{2}$ : C, 66.65; H, 4.07; N, 21.20; $\mathrm{S}, 8.09$. Found: $\mathrm{C}, 66.93 ; \mathrm{H}, 4.15 ; \mathrm{N}$, 21.10; S, 8.15.

2,3-Bis((5,6-Dihydro-6-(4-flourophenyl)-14H-indolo[2,3-d]-[1,2,4-triazolo][4,3-b]pyridazin-3-ylsulfanyl)methyl)quinoxaline 20. Yield: 87\%; m.p. $294_{\text {decomp }}{ }^{\circ} \mathrm{C} ;{ }^{1} \mathrm{H}-\mathrm{NMR}$ (DMSO- $\left.d_{6}, 300 \mathrm{MHz}\right) \delta 5.045\left(\mathrm{~s}, 4 \mathrm{H}, 2 \mathrm{SCH}_{2}\right), 5.92$ (d, 2 $\mathrm{H}, J 6.0 \mathrm{~Hz}, 2 \mathrm{H}-6$ Pyridazine), 7.03 to $7.11(\mathrm{~m}, 6 \mathrm{H}), 7.22(\mathrm{dd}, 2 \mathrm{H}, J$ 7.2, J $8.1 \mathrm{~Hz}), 7.29$ to $7.38(\mathrm{~m}, 8 \mathrm{H}), 7.47$ (d, $2 \mathrm{H}, J 8.1 \mathrm{~Hz}), 7.80$ to $7.83(\mathrm{~m}, 2 \mathrm{H}), 7.98$ to $8.02(\mathrm{~m}, 2 \mathrm{H}), 12.36$ (br. s, $\left.2 \mathrm{H}, 2 \mathrm{NH}_{\text {Indole }}\right) ;{ }^{13} \mathrm{C}-\mathrm{NMR}$ (DMSO- $\left.d_{6}, 75 \mathrm{MHz}\right) \delta 35.84\left(2 \mathrm{SCH}_{2}\right), 55.92$ (2 C-6Pyridazine), 112.89, 114.28, 119.87, 120.80, 121.60, $123.99,124.88,128.71,129.75,129.84,130.74,136.51,137.92,140.51,146.47,149.03,151.10 ; \operatorname{IR}\left(\mathrm{cm}^{-1}\right)$ : 1608, 3060, 3147; HRMS (FAB +ve) calculated for $\mathrm{C}_{44} \mathrm{H}_{31} \mathrm{~N}_{12} \mathrm{~S}_{2} \mathrm{~F}_{2}\left(\mathrm{M}^{+}\right)$: 829.2204. Found: 829.2220.

2,3-Bis((6-(3-Bromophenyl)-5,6-dihydro-14H-indolo[2,3-d]-[1,2,4-triazolo][4,3-b]pyridazin-3-ylsulfanyl)methyl)quinoxaline 21. Yield: $79 \%$; m.p. $298_{\text {decomp. }}{ }^{\circ} \mathrm{C} ;{ }^{1} \mathrm{H}-\mathrm{NMR}$ (DMSO- $\left.d_{6}, 300 \mathrm{MHz}\right) \delta 5.05\left(\mathrm{~s}, 4 \mathrm{H}, 2 \mathrm{SCH}_{2}\right), 5.99(\mathrm{~d}, 2 \mathrm{H}$, $\left.J 5.7 \mathrm{~Hz}, 2 \mathrm{H}-6_{\text {Pyridazine }}\right), 7.09$ (dd, $\left.2 \mathrm{H}, J 7.8, J 7.5 \mathrm{~Hz}\right), 7.18$ to $7.52(\mathrm{~m}, 16 \mathrm{H}), 7.80$ to $7.83(\mathrm{~m}, 2 \mathrm{H}), 7.98$ to $8.02(\mathrm{~m}, 2 \mathrm{H}), 12.39$ (br. s, $\left.2 \mathrm{H}, 2 \mathrm{NH}_{\text {Indole }}\right) ;{ }^{13} \mathrm{C}-\mathrm{NMR}$ (DMSO- $\left.d_{6}, 75 \mathrm{MHz}\right) \delta 35.36\left(2 \mathrm{SCH}_{2}\right), 55.22(2$ C-6 Pyridazine), 112.44, 113.27, 119.34, 120.45, 121.06, 121.65, 123.60, 124.38, 128.23, 129.89, 130.24, 130.48, 130.60, 137.37, 140.00, 142.74, 145.87, 148.62, 150.53; IR ( $\left.\mathrm{cm}^{-1}\right)$ : 1609, 2924, 3058; HRMS (FAB +ve) calculated for $\mathrm{C}_{44} \mathrm{H}_{31} \mathrm{~N}_{12} \mathrm{~S}_{2} \mathrm{Br}_{2}\left(\mathrm{M}^{+}\right)$: 949.0603 . Found: 949.0615 .

2,3-Bis((5,6-Dihydro-14H-indolo[2,3-d]-6-p-tolyl-[1,2,4-triazolo][4,3-b]pyridazin-3-ylsulfanyl)methyl)quinoxaline 22. Yield: 78\%; m.p. 284 to $285{ }^{\circ} \mathrm{C} ;{ }^{1} \mathrm{H}-\mathrm{NMR}$ (DMSO- $\left.d_{6}, 300 \mathrm{MHz}\right) \delta 2.18\left(\mathrm{~s}, 6 \mathrm{H}, 2 \mathrm{CH}_{3}\right), 5.02(\mathrm{~s}, 4 \mathrm{H}$, $\left.2 \mathrm{SCH}_{2}\right), 5.83\left(\mathrm{~d}, 2 \mathrm{H}, J 6.6 \mathrm{~Hz}, 2 \mathrm{H}-6_{\text {Pyridazine }}\right), 7.00$ to $7.32(\mathrm{~m}, 16 \mathrm{H}), 7.46(\mathrm{~d}, 2 \mathrm{H}, J 8.4 \mathrm{~Hz}), 7.80$ to $7.83(\mathrm{~m}, 2 \mathrm{H}), 7.99-8.01$ (m, $2 \mathrm{H}), 12.31$ (br. s, $2 \mathrm{H}, 2 \mathrm{NH}_{\text {Indole }}$ ); ${ }^{13} \mathrm{C}-\mathrm{NMR}$ (DMSO-d $\left.d_{6}, 75 \mathrm{MHz}\right) 21.08$ (2 $\left.\mathrm{CH}_{3}\right), 35.79\left(2 \mathrm{SCH}_{2}\right), 56.52$ (2 C-6 Pyridazine $), 112.82,114.67,119.93,120.65,121.58,123.88,124.93,127.75$, 128.72, 129.36, 130.72, 137.20, 137.35, 137.91, 140.53, 146.56, 148.97, 151.14; IR (cm $\left.{ }^{-1}\right):$ 1610, 2917; HRMS (FAB +ve) calculated for $\mathrm{C}_{46} \mathrm{H}_{37} \mathrm{~N}_{12} \mathrm{~S}_{2}\left(\mathrm{M}^{+}\right)$: 821.2767. Found: 821.2767.

2,3-Bis((5,6-Dihydro-14H-indolo[2,3-d]-6-(2,3,4-trimethoxyphenyl)-[1,2,4-triazolo][4,3-b]pyridazin-3-ylsulfanyl) methyl)quinoxaline 23. Yield: $90 \%$; m.p. 280 to $283{ }^{\circ} \mathrm{C} ;{ }^{1} \mathrm{H}-\mathrm{NMR}$ (DMSO-d ${ }_{6}, 400 \mathrm{MHz}$ ) $\delta 3.71,3.78,3.92$ (3s, $\left.18 \mathrm{H}, 6 \mathrm{OCH}_{3}\right), 4.98\left(\mathrm{~s}, 4 \mathrm{H}, 2 \mathrm{SCH}_{2}\right), 6.02\left(\mathrm{~d}, 2 \mathrm{H}, \mathrm{J} 8.0 \mathrm{~Hz}, 2 \mathrm{H}-6_{\text {Pyridazine }}\right), 6.48$ (d, $\left.2 \mathrm{H}, \mathrm{J} 8.4 \mathrm{~Hz}\right)$, $6.64(\mathrm{~d}, 2 \mathrm{H}, \mathrm{J} 8.4 \mathrm{~Hz}), 6.97(\mathrm{br}, 4 \mathrm{H}), 7.11(\mathrm{~d}, 2 \mathrm{H}, \mathrm{J} 8.4 \mathrm{~Hz}), 7.18(\mathrm{br}, 2 \mathrm{H}), 7.45(\mathrm{~d}, 2 \mathrm{H}, \mathrm{J}$ 8.0 Hz), 7.78 (br, 2 H), 7.93 (br, $2 \mathrm{H}), 12.34$ (br. s, $\left.2 \mathrm{H}, 2 \mathrm{NH}_{\text {Indole }}\right) ;{ }^{13} \mathrm{C}-\mathrm{NMR}\left(\mathrm{DMSO}_{6}, \mathrm{~d}_{6}, 100 \mathrm{MHz}\right) \delta 35.15\left(2 \mathrm{SCH}_{2}\right), 51.60$, 
55.71, 60.37, 61.51 (2 C-6 Pyridazin, $\left.6 \mathrm{OCH}_{3}\right), 107.61,112.33,113.40,119.21,119.98,121.89,123.07,123.34$, 123.81, 124.41, 128.17, 130.23, 137.57, 140.02, 141.87, 146.22, 148.23, 150.68, 151.58, 153.39; IR (cm $\left.{ }^{-1}\right)$ : 1605, 2931, 3199; HRMS (FAB +ve) calculated for $\mathrm{C}_{50} \mathrm{H}_{45} \mathrm{~N}_{12} \mathrm{O}_{6} \mathrm{~S}_{2}\left(\mathrm{M}^{+}\right)$: 973.3026. Found: 973.3053.

2,3-Bis((6-(4-Chlorophenyl)-5,6-dihydro-14H-indolo[2,3-d]-[1,2,4-triazolo][4,3-b]pyridazin-3-ylsulfanyl) methyl)quinoxaline 24. Yield: 77\%; m.p. $294_{\text {decomp. }}{ }^{\circ} \mathrm{C} ;{ }^{1} \mathrm{H}-\mathrm{NMR}\left(\mathrm{DMSO}-\mathrm{d}_{6}, 300 \mathrm{MHz}\right) \delta 5.05$ (s, $4 \mathrm{H}, 2$ $\left.\mathrm{SCH}_{2}\right), 5.95$ (d, $\left.2 \mathrm{H}, \mathrm{J} 6.0 \mathrm{~Hz}, 2 \mathrm{H}-6_{\text {Pyridazine }}\right), 7.07$ (dd, $\left.2 \mathrm{H}, \mathrm{J} 7.5 \mathrm{~Hz}\right), 7.20$ to 7.48 (m, $\left.16 \mathrm{H}\right), 7.80$ to 7.84 (m, $2 \mathrm{H}), 7.98$ to 8.01 (m, $2 \mathrm{H}), 12.37$ (br. s, $\left.2 \mathrm{H}, 2 \mathrm{NH}_{\text {Indol }}\right) ;{ }^{13} \mathrm{C}-\mathrm{NMR}\left(\mathrm{DMSO}-\mathrm{d}_{6}, 75 \mathrm{MHz}\right) 35.81$ (2 $\left.\mathrm{SCH}_{2}\right), 55.76$ (2 C-6 Pyridazine $), 112.90,113.98,119.86,120.85,121.61,124.90,128.71,128.84,129.57,130.75$, 132.76, 137.91, 139.44, 140.50, 146.41, 149.06, 151.10; IR $\left(\mathrm{cm}^{-1}\right)$ : 1600, 2927, 3205; HRMS (FAB +ve) calculated for $\mathrm{C}_{44} \mathrm{H}_{31} \mathrm{~N}_{12} \mathrm{~S}_{2} \mathrm{Cl}_{2}\left(\mathrm{M}^{+}\right)$: 861.1613. Found: 861.1607.

2,3-Bis((6-(4-Bromophenyl)-5,6-dihydro-14H-indolo[2,3-d]-[1,2,4-triazolo][4,3-b]pyridazin-3-ylsulfanyl)methyl) quinoxaline 25. Yield: $81 \%$; m.p. 284 to $285{ }^{\circ} \mathrm{C}$; ${ }^{1} \mathrm{H}-\mathrm{NMR}$ (DMSO- $\left.d_{6}, 300 \mathrm{MHz}\right) \delta 5.05\left(\mathrm{~s}, 4 \mathrm{H}, 2 \mathrm{SCH}_{2}\right)$, $5.94\left(\mathrm{~d}, 2 \mathrm{H}, J 5.7 \mathrm{~Hz}, 2 \mathrm{H}-6_{\text {Pyridazine }}\right), 7.08$ (dd, $\left.2 \mathrm{H}, J 7.5 \mathrm{~Hz}\right), 7.21$ to 7.49 (m, $\left.16 \mathrm{H}\right), 7.80$ to 7.84 (m, 2 $\mathrm{H}), 7.98$ to 8.01 (m, $2 \mathrm{H}), 12.37$ (br. s, $\left.2 \mathrm{H}, 2 \mathrm{NH}_{\text {Indole }}\right) ;{ }^{13} \mathrm{C}-\mathrm{NMR}$ (DMSO-d 6 , $\left.75 \mathrm{MHz}\right) 35.82\left(2 \mathrm{SCH}_{2}\right)$, 55.80 (2 C-6Pyridazin), 112.91, 113.92, 119.86, 120.86, 121.35, 124.04, 124.91, 128.71, 129.93, 130.74, 131.77, 137.92, 139.88, 140.51, 146.41, 149.08, 151.09; IR (cm $\left.{ }^{-1}\right):$ 1602, 2925, 3198; HRMS (FAB +ve) calculated for $\mathrm{C}_{44} \mathrm{H}_{31} \mathrm{~N}_{12} \mathrm{~S}_{2} \mathrm{Br}_{2}\left(\mathrm{M}^{+}\right)$: 949.0603 . Found: 949.0669 .

\section{Conclusions}

A fascinating cyclization reaction approach was achieved when 4-amino-5-(1H-indol-2-yl)-2,4dihydro-3H-1,2,4-triazole-3-thione 1 was reacted with ten aromatic aldehydes in ethanol and concentrated $\mathrm{HCl}$ to obtain indolo-triazolo-pyridazinethiones 7-16 in an excellent yield. Moreover, the alkylation of indolo-triazolo-pyridazinethiones 7-13 with 2,3-bis(bromomethyl)quinoxaline as a linker in acetone and $\mathrm{K}_{2} \mathrm{CO}_{3}$ afforded 2,3-bis((5,6-dihydro-14H-indolo[2,3- $d$ ]-6-aryl-[1,2,4-triazolo][4,3-b]pyridazin-3 ylsulfanyl)methyl)quinoxalines 19-25 in a high yield. Studies to establish their applications are in progress.

Supplementary Materials: NMR spectra (Figures S1-S44); and IR analyses (Figures S45-S60) associated with this article can be found in the online version.

Author Contributions: Conceptualization, A.T.A.B.; Data curation, A.A.M.S.; Formal analysis, S.Y. and A.B.; Funding acquisition, A.B.; Methodology, A.T.A.B.; Software, S.Y.; Supervision, A.T.A.B.; Validation, A.A.M.S.; Visualization, A.B.; Writing-Original draft, A.T.A.B.; Writing-Review and editing, A.B. All authors have read and agreed to the published version of the manuscript.

Funding: The authors would like to extend their sincere appreciation to the Researchers Supporting project number (RSP-2019/64), King Saud University, Riyadh, Saudi Arabia.

Acknowledgments: The authors would like to extend their sincere appreciation to the Researchers Supporting project number (RSP-2019/64), King Saud University, Riyadh, Saudi Arabia.

Conflicts of Interest: The authors declare no conflict of interest.

\section{References}

1. Kumari, A.; Singh, R.K. Medicinal chemistry of indole derivatives: Current to future therapeutic prospectives. Bioorg. Chem. 2019, 89, 103021. [CrossRef] [PubMed]

2. Kharb, R.; Sharma, P.C.; Yar, M.S. Pharmacological significance of triazole scaffold. J. Enzyme Inhib. Med. Chem. 2011, 26, 1-21. [CrossRef] [PubMed]

3. Kashyap, A.; Silakari, O. Triazoles: Multidimensional 5-membered nucleus for designing multitargeting agents. In Key Heterocycle Cores for Designing Multitargeting Molecules; Elsevier: Amsterdam, The Netherlands, 2018; pp. 323-342.

4. Maftei, C.V.; Fodor, E.; Jones, P.G.; Daniliuc, C.G.; Franz, M.H.; Kelter, G.; Fiebig, H.-H.; Tamm, M.; Neda, I. Novel 1,2,4-oxadiazoles and trifluoromethylpyridines related to natural products: Synthesis, structural analysis and investigation of their antitumor activity. Tetrahedron 2016, 72, 1185-1199. [CrossRef] 
5. Neda, I.; Kaukorat, T.; Schmutzler, R.; Niemeyer, U.; Kutscher, B.; Pohl, J.; Engel, J. Benzodiaza-, Benzoxaza- and benzodioxaphosphorinones- formation, reactivity, structure and biological activity. Phosphorus Sulfur Silicon 2000, 162, 81-218. [CrossRef]

6. Simon, M.; Csunderlik, C.; Cotarca, L.; Caproiu, M.T.; Neda, I.; Turoczi, M.C.; Volpicelli, R. Synthesis of new active 0-nitrophenyl carbamates. Synth. Comun. 2005, 35, 1471-1479. [CrossRef]

7. Mohammad, Y.; Fazili, K.M.; Bhat, K.A.; Ara, T. Synthesis and biological evaluation of novel 3-O-tethered triazoles of diosgenin as potent antiproliferative agents. Steroids 2017, 118, 1-8.

8. Huang, M.; Deng, Z.; Tian, J.; Liu, T. Synthesis and biological evaluation of salinomycin triazole analogues as anticancer agents. Eur. J. Med. Chem. 2017, 127, 900-908. [CrossRef]

9. Gujjar, R.; Marwaha, A.; El Mazouni, F.; White, J.; White, K.L.; Creason, S.; Shackleford, D.M.; Baldwin, J.; Charman, W.N.; Buckner, F.S.; et al. Identification of a metabolically stable triazolopyrimidine-based dihydroorotate dehydrogenase inhibitor with antimalarial activity in mice. J. Med. Chem. 2009, 52, 1864-1872. [CrossRef]

10. Chen, M.; Lu, S.; Yuan, G.; Yang, S.; Du, X. Synthesis and antibacterial activity of some heterocyclic $\beta$-enamino ester derivatives with 1, 2, 3-triazole. Heterocycl. Commun. 2000, 6, 421-426. [CrossRef]

11. Ayati, A.; Emami, S.; Foroumadi, A. The importance of triazole scaffold in the development of anticonvulsant agents. Eur. J. Med. Chem. 2016, 109, 380-392. [CrossRef]

12. Akhtar, T.; Hameed, S.; Khan, K.M.; Choudhary, M.I. Syntheses, urease inhibition, and antimicrobial studies of some chiral 3-substituted-4-amino-5-thioxo-1H, 4H-1,2,4-triazoles. Med. Chem. 2008, 4, 539-543. [CrossRef]

13. Sevaille, L.; Gavara, L.; Bebrone, C.; De Luca, F.; Nauton, L.; Achard, M.; Mercuri, P.; Tanfoni, S.; Borgianni, L.; Guyon, C.; et al. 1,2,4-Triazole-3-thione compounds as inhibitors of dizinc metallo- $\beta$-lactamases. ChemMedChem 2017, 12, 972-985. [CrossRef]

14. Gilmore, J.L.; King, B.W.; Asakawa, N.; Harrison, K.; Tebben, A.; Sheppeck, J.E., II; Liu, R.Q.; Covington, M.; Duan, J.J.W. Synthesis and structure-activity relationship of a novel, non-hydroxamate series of TNF- $\alpha$ converting enzyme inhibitors. Bioorg. Med. Chem. Lett. 2007, 17, 4678-4682. [CrossRef] [PubMed]

15. Maingot, L.; Leroux, F.; Landry, V.; Dumont, J.; Nagase, H.; Villoutreix, B.; Sperandio, O.; Deprez-Poulain, R.; Deprez, B. New non-hydroxamic ADAMTS-5 inhibitors based on the 1,2,4-triazole-3-thiol scaffold. Bioorg. Med. Chem. Lett. 2010, 20, 6213-6216. [CrossRef] [PubMed]

16. Kruse, L.I.; Kaiser, C.; DeWolf, W.E.; Finkelstein, J.A.; Frazee, J.S.; Hilbert, E.L.; Ross, S.T.; Flaim, K.E.; Sawyer, J.L. Some benzyl-substituted imidazoles, triazoles, tetrazoles, pyridinethiones, and structural relatives as multisubstrate inhibitors of dopamine. $\beta$-hydroxylase. 4 . Structure-activity relationships at the copper binding site. J. Med. Chem. 1990, 33, 781-789. [CrossRef] [PubMed]

17. Timur, İ.; Kocyigit, Ü.M.; Dastan, T.; Sandal, S.; Ceribas1, A.O.; Taslimi, P.; Gulcin, İ.; Koparir, M.; Karatepe, M.; Çiftçi, M. In vitro cytotoxic and in vivo antitumoral activities of some aminomethyl derivatives of 2, 4-dihydro-3H-1,2,4-triazole-3-thiones-Evaluation of their acetylcholinesterase and carbonic anhydrase enzymes inhibition profiles. J. Biochem. Mol. Toxic. 2019, 33, e22239. [CrossRef]

18. Hamdy, R.; Ziedan, N.; Ali, S.; El-Sadek, M.; Lashin, E.; Brancale, A.; Jones, A.T.; Westwell, A.D. Synthesis and evaluation of 3-(benzylthio)-5-(1H-indol-3-yl)-1,2,4-triazol-4-amines as Bcl-2 inhibitory anticancer agents. Bioorg. Med. Chem. Lett. 2013, 23, 2391-2394. [CrossRef]

19. Ziedan, N.I.; Hamdy, R.; Cavaliere, A.; Kourti, M.; Prencipe, F.; Brancale, A.; Jones, A.T.; Westwell, A.D. Virtual screening, SAR, and discovery of 5-(indole-3-yl)-2-[(2-nitrophenyl) amino][1,3,4]-oxadiazole as a novel Bcl-2 inhibitor. Chem. Biol. Drug Des. 2017, 90, 147-155. [CrossRef]

20. Boraei, A.T.; Ghabbour, H.A.; Gomaa, M.S.; El Ashry, E.S.H.; Barakat, A. Synthesis and anti-proliferative assessment of triazolo-thiadiazepine and triazolo-thiadiazine scaffolds. Molecules 2019, 24, 4471. [CrossRef]

21. Boraei, A.T.A.; Gomaa, M.S.; El Sayed, E.S.H.; Duerkop, A. Design, selective alkylation and X-ray crystal structure determination of dihydro-indolyl-1,2,4-triazole-3-thione and its 3-benzylsulfanyl analogue as potent anticancer agents. Eur. J. Med. Chem. 2017, 125, 360-371. [CrossRef]

22. Darestani-Farahani, M.; Faridbod, F.; Ganjali, M.R. A sensitive fluorometric DNA nanobiosensor based on a new fluorophore for tumor suppressor gene detection. Talanta 2018, 190, 140-146. [CrossRef]

23. Shi, Z.; Zhao, Z. Microwave irradiation synthesis of novel indole triazole Schiff base fluorescent probe for Al3+ ion. Inorganica Chim. Acta 2019, 498, 119135. [CrossRef] 
24. Boraei, A.T.; Singh, P.K.; Sechi, M.; Satta, S. Discovery of novel functionalized 1, 2, 4-triazoles as PARP-1 inhibitors in breast cancer: Design, synthesis and antitumor activity evaluation. Eur. J. Med. Chem. 2019, 182, 111621. [CrossRef] [PubMed]

25. Boraei, A.T.; Ashour, H.K.; El Sayed, H.; Abdelmoaty, N.; El-Falouji, A.I.; Gomaa, M.S. Design and synthesis of new phthalazine-based derivatives as potential EGFR inhibitors for the treatment of hepatocellular carcinoma. Bioorg. Chem. 2019, 85, 293-307. [CrossRef]

26. Chehrouri, M.; Othman, A.A.; Jiménez-Cecilia, S.; Moreno-Cabrerizo, C.; Sansano, J.M. 4-Amino-3-pentadecyl-3H-1,2,4-triazole-3-thiones and 3-pentadecyl-1,3,4-oxadiazole-2 (3H)-thione for the preparation of dimeric palladium (II) complexes and their applications in Tsuji-Trost and Mizoroki-Heck reactions. Synth. Commun. 2019, 49, 1301-1307. [CrossRef]

27. Abdallah, M.A.; Riyadh, S.M.; Abbas, I.M.; Gomha, S.M. Synthesis and biological activities of 7-arylazo-7H-pyrazolo [5,1-c][1,2,4] triazol-6 (5H)-ones and 7-arylhydrazono-7H-[1,2,4] triazolo [3,4- $b][1,3,4]$ thiadiazines. J. Chin. Chem. Soc. 2005, 52, 987-994. [CrossRef]

28. Gomha, S.M.; Riyadh, S.M. Synthesis under microwave irradiation of $[1,2,4]$ triazolo $[3,4-b][1,3,4]$ thiadiazoles and other diazoles bearing indole moieties and their antimicrobial evaluation. Molecules 2011, 16, 8244-8256. [CrossRef]

29. Peng, Y.; Zhao, Z.; Liu, X.; Li, G. Microwave-assisted synthesis and biological activity of new Schiff bases derived from dimers of 4-amino-3-[3-(1-benzyl)indole]-5-thiomethyl-1,2,4-triazole. Res. Chem. Intermediat. 2013, 39, 1897-1905. [CrossRef]

30. Peng, Y.L.; Liu, X.L.; Wang, X.H.; Zhao, Z.G. Microwave-assisted synthesis and antibacterial activity of derivatives of 3-[1-(4-fluorobenzyl)-1H-indol-3-yl]-5-(4-fluorobenzylthio)-4H-1, 2, 4-triazol-4-amine. Chem. Pap. 2014, 68, 401-408. [CrossRef]

31. Shi, Z.; Zhao, Z.; Huang, M.; Fu, X. Ultrasound-assisted, one-pot, three-component synthesis and antibacterial activities of novel indole derivatives containing 1,3,4-oxadiazole and 1,2,4-triazole moieties. C. R. Chim. 2015, 18, 1320-1327. [CrossRef]

32. Cascioferro, S.; Parrino, B.; Li Petri, G.; Cusumano, M.G.; Schillaci, D.; Sarno, V.D.; Musella, S.; Giovannetti, E.; Cirrincione, G.; Diana, P. 2,6-Disubstituted imidazo[2,1-b][1,3,4]thiadiazole derivatives as potent staphylococcal biofilm inhibitors. Eur. J. Med. Chem. 2019, 167, 200-210. [CrossRef]

33. Ishikawa, H.; Sugiyama, T.; Kurita, K.; Yokoyama, A. Synthesis and antimicrobial activity of 2, 3-bis (bromomethyl) quinoxaline derivatives. Bioorg. Chem. 2012, 41, 1-5. [CrossRef] [PubMed]

34. Parrino, B.; Carbone, A.; Spanò, V.; Montalbano, A.; Giallombardo, D.; Barraja, P.; Attanzio, A.; Tesoriere, L.; Sissi, C.; Palumbo, M.; et al. Aza-isoindolo and isoindolo-azaquinoxaline derivatives with antiproliferative activity. Eur. J. Med. Chem. 2015, 94, 367-377. [CrossRef] [PubMed]

35. Parrino, B.; Carbone, A.; Ciancimino, C.; Spanò, V.; Montalbano, A.; Barraja, P.; Cirrincione, G.; Diana, P.; Sissi, C.; Palumbo, M.; et al. Water-soluble isoindolo[2,1-a]quinoxalin-6-imines: In vitro antiproliferative activity and molecular mechanism(s) of action. Eur. J. Med. Chem. 2015, 94, 149-162. [CrossRef] [PubMed]

36. Bruker, A. SAINT Software Reference Manual; Bruker AXS, Inc.: Madison, WI, USA, 1998.

37. Spek, L.A. Single-crystal structure validation with the program PLATON. J. Appl. Chem. 2002, 36, 7-13.

38. Sheldrick, G.M. Crystal structure refinement with SHELXL. Acta Cryst. C. 2015, 71, 3-8. [CrossRef] [PubMed]

Sample Availability: Samples of the compounds 2-16 and 19-25 are available from the authors. 